TITLE:

\title{
Quantum-mechanical generation of gravitational waves in a braneworld
}

$\operatorname{AUTHOR}(\mathrm{S})$ :

Kobayashi, T; Tanaka, T

CITATION:

Kobayashi, T ...[et al]. Quantum-mechanical generation of gravitational waves in a braneworld. PHYSICAL REVIEW D 2005, 71(12): 124028.

ISSUE DATE:

2005-06

URL:

http://hdl.handle.net/2433/49922

RIGHT:

Copyright 2005 American Physical Society 
PHYSICAL REVIEW D 71, 124028 (2005)

\title{
Quantum-mechanical generation of gravitational waves in a braneworld
}

\author{
Tsutomu Kobayashi* and Takahiro Tanaka ${ }^{\dagger}$ \\ Department of Physics, Kyoto University, Kyoto 606-8502, Japan \\ (Received 16 May 2005; published 17 June 2005)
}

\begin{abstract}
We study the quantum-mechanical generation of gravitational waves during inflation on a brane embedded in a five-dimensional anti de Sitter bulk. To make the problem well posed, we consider the setup in which both initial and final phases are given by a de Sitter brane with different values of the Hubble expansion rate. Assuming that the quantum state is in a de Sitter invariant vacuum in the initial de Sitter phase, we numerically evaluate the amplitude of quantum fluctuations of the growing solution of the zero mode in the final de Sitter phase. We find that the vacuum fluctuations of the initial Kaluza-Klein gravitons as well as of the zero mode gravitons contribute to the final amplitude of the zero mode on small scales, and the power spectrum is quite well approximated by what we call the rescaled spectrum, which is obtained by rescaling the standard four-dimensional calculation following a simple mapping rule. Our results confirm the speculation raised in Ref. [T. Kobayashi, H. Kudoh, and T. Tanaka, Phys. Rev. D 68, 044025 (2003).] before.
\end{abstract}

DOI: $10.1103 /$ PhysRevD.71.124028

PACS numbers: $04.50 .+\mathrm{h}, 11.10 . \mathrm{Kk}, 98.80 . \mathrm{Cq}$

\section{INTRODUCTION}

In recent years there has been a growing attention to braneworld scenarios, in which our observable Universe is realized as a brane embedded in a higher dimensional bulk spacetime [1]. Among various models a simple setup called the Randall-Sundrum type II model [2] is of particular interest because, despite gravity propagating into an infinite extra dimension, four-dimensional general relativity can be recovered on the brane due to the warped geometry of anti de Sitter (AdS) spacetime [2-4]. Cosmological consequences of the Randall-Sundrum type braneworld have been widely discussed [5-10], especially regarding cosmological perturbations [11].

The generation and evolution of perturbations are among the most important issues in cosmology because of their direct link to cosmological observations such as the stochastic gravitational wave background and the temperature anisotropy of the cosmic microwave background (CMB), by which we can probe the early Universe. While the cosmological perturbation theory in the conventional four-dimensional Universe is rather established [12,13], calculating cosmological perturbations in the braneworld still remains a difficult problem. Although some attempts have been made concerning scalar perturbations [14-19], further progress is awaited to give a clear prediction about the CMB anisotropy in the Randall-Sundrum braneworld. Almost the same is true for gravitational wave (tensor) perturbations $[8,20-31]$. However, since their generation and evolution depend basically only on the background geometry, they are slightly easier to handle.

During inflation superhorizon gravitational wave perturbations are generated from vacuum fluctuations of gravitons. The pure de Sitter braneworld is the special case that

\footnotetext{
*Electronic address: tsutomu@tap.scphys.kyoto-u.ac.jp

†Electronic address: tama@scphys.kyoto-u.ac.jp
}

allows definite analytical computation of quantum fluctuations. Thanks to the symmetry of the de Sitter group, the perturbation equation becomes separable and can be solved exactly [20]. The time variation of the Hubble parameter generally causes mixing of a massless zero mode and massive Kaluza-Klein modes, and this effect was investigated based on the "junction" models, which assume an instantaneous transition from a de Sitter to a Minkowski brane [21] or to another de Sitter brane [22]. These works discussed the quantum-mechanical generation of gravitational waves within such limited and simplified models.

As for the classical evolution of gravitational waves during the radiation dominated epoch, several studies have been done [23-26], assuming that a given single initial mode for each comoving wave number dominates. The focus of these works is mainly on the evolution of modes which reenter the horizon in the high-energy regime, while the late time evolution is worked out analytically in Refs. $[17,31]$ by resorting to low-energy approximation methods.

In this paper we consider the generation of primordial gravitational waves during inflation in more general models of the Randall-Sundrum type. For definiteness, we adopt a simple setup in which both initial and final phases are described by de Sitter braneworlds. Two de Sitter phases with different values of the Hubble expansion rate are smoothly interpolated. This work is an extension of the work on the junction models by the present authors in collaboration with H. Kudoh [22]. In the previous analysis the transition of the Hubble rate was abrupt and the gap was assumed to be infinitesimal. Here we extend the previous results to more general models with smooth transition by using numerical calculations with a refined formulation.

This paper is organized as follows. In the next section we briefly summarize past studies [20-22] on the generation of gravitational waves via quantum fluctuations during inflation in the Randall-Sundrum braneworld, emphasizing 
the mapping formula introduced in Ref. [22]. In Sec. III we describe our numerical scheme to investigate the generation of gravitational waves, and then in Sec. IV we present results of our calculations. Section V is devoted to discussion.

\section{GRAVITATIONAL WAVES IN INFLATIONARY BRANEWORLD}

\section{A. Pure de Sitter brane}

The background spacetime that we consider is composed of a five-dimensional AdS bulk, whose metric is given in the Poincare coordinates by

$$
d s^{2}=\frac{\ell^{2}}{z^{2}}\left(-d t^{2}+\delta_{i j} d x^{i} d x^{j}+d z^{2}\right),
$$

and a Friedmann brane at $z=z(t)$. Here $\ell$ is the bulk curvature length, which is constrained by tabletop experiments as $\ell \lesssim 0.1 \mathrm{~mm}$ [32].

First let us consider the generation of gravitational waves from pure de Sitter inflation on the brane [20]. The coordinate system appropriate for the present situation is

$$
d s^{2}=\frac{\ell^{2}}{\sinh ^{2} \xi}\left[\frac{1}{\eta^{2}}\left(-d \eta^{2}+\delta_{i j} d x^{i} d x^{j}\right)+d \xi^{2}\right],
$$

which is obtained from Eq. (1) by a coordinate transformation

$$
\begin{gathered}
t=\eta \cosh \xi+t_{0}, \\
z=-\eta \sinh \xi,
\end{gathered}
$$

where $t_{0}$ is an arbitrary constant and $\eta$ is the conformal time, which is negative. The de Sitter brane is located at

$$
\xi=\xi_{b}=\text { constant }
$$

and the Hubble parameter on the brane is given by

$$
H=\ell^{-1} \sinh \xi_{b} .
$$

The gravitational wave perturbations are described by the metric

$$
d s^{2}=\frac{\ell^{2}}{\sinh ^{2} \xi}\left\{\frac{1}{\eta^{2}}\left[-d \eta^{2}+\left(\delta_{i j}+h_{i j}\right) d x^{i} d x^{j}\right]+d \xi^{2}\right\},
$$

and we decompose the perturbations into the spatial Fourier modes as

$$
h_{i j}=\frac{\sqrt{2}}{\left(2 \pi M_{5}\right)^{3 / 2}} \int d^{3} k \phi_{\mathbf{k}}(\eta, \xi) e^{i \mathbf{k} \cdot \mathbf{x}} e_{i j},
$$

where $e_{i j}$ is the transverse-traceless polarization tensor and $M_{5}$ is the fundamental mass scale which is related to the four-dimensional Planck mass $M_{\mathrm{Pl}}$ by $\ell\left(M_{5}\right)^{3}=M_{\mathrm{Pl}}^{2}$. The prefactor $\sqrt{2} /\left(M_{5}\right)^{3 / 2}$ is chosen so that the kinetic term for $\phi_{\mathbf{k}}$ in the effective action is canonically normalized. Hereafter we will suppress the subscript $\mathbf{k}$. The linearized Einstein equations give the following Klein-Gordon-type equation for $\phi$ :

$$
\left[\frac{\partial^{2}}{\partial \eta^{2}}-\frac{2}{\eta} \frac{\partial}{\partial \eta}+k^{2}-\frac{\sinh ^{3} \xi}{\eta^{2}} \frac{\partial}{\partial \xi} \frac{1}{\sinh ^{3} \xi} \frac{\partial}{\partial \xi}\right] \phi=0,
$$

which is obviously separable. Assuming the $Z_{2}$ symmetry across the brane, the boundary condition on the brane is given by

$$
\left.\left.n^{\mu} \partial_{\mu} \phi\right|_{\text {brane }} \propto \partial_{\xi} \phi\right|_{\xi=\xi_{b}}=0,
$$

where $n^{\mu}$ is a unit normal to the brane. Equation (9) admits one discrete zero mode,

$$
\phi_{0}(\eta)=\mathcal{N} \cdot \ell^{-1 / 2} \frac{H}{\sqrt{2 k}}\left(\eta-\frac{i}{k}\right) e^{-i k \eta},
$$

where $\mathcal{N}$ is a normalization constant, which will be fixed later, as well as massive Kaluza-Klein (KK) modes,

$$
\phi_{\nu}(\eta, \xi)=\psi_{\nu}(\eta) \chi_{\nu}(\xi),
$$

where $\psi_{\nu}(\eta)$ and $\chi_{\nu}(\xi)$ obey

$$
\begin{aligned}
& {\left[\frac{\partial^{2}}{\partial \eta^{2}}-\frac{2}{\eta} \frac{\partial}{\partial \eta}+k^{2}+\frac{\nu^{2}+9 / 4}{\eta^{2}}\right] \psi_{\nu}(\eta)=0,} \\
& {\left[\sinh ^{3} \xi \frac{\partial}{\partial \xi} \frac{1}{\sinh ^{3} \xi} \frac{\partial}{\partial \xi}+\nu^{2}+\frac{9}{4}\right] \chi_{\nu}(\xi)=0,}
\end{aligned}
$$

and the corresponding Kaluza-Klein mass is given by

$$
m^{2}=\left(\nu^{2}+\frac{9}{4}\right) H^{2} \geq \frac{9}{4} H^{2} .
$$

Because of the mass gap $\Delta m=3 H / 2$ [7] between the zero mode and the lowest KK mode, all the massive KK modes decay on the brane at superhorizon scales during inflation. The explicit forms of the KK mode functions are shown in the Appendix.

In inflationary cosmology, fluctuations in the graviton field (and other fields such as the inflaton) are considered to be generated quantum-mechanically. Following the standard canonical quantization scheme we quantize the graviton field. Treating $\phi$ as an operator, it may be expanded as

$$
\phi=\hat{a}_{0} \phi_{0}+\hat{a}_{0}^{\dagger} \phi_{0}^{*}+\int_{0}^{\infty} d \nu\left(\hat{a}_{\nu} \phi_{\nu}+\hat{a}_{\nu}^{\dagger} \phi_{\nu}^{*}\right) .
$$

The annihilation and creation operators $\hat{a}_{n}$ and $\hat{a}_{n}^{\dagger}$ with $n=0$ or $\nu$ satisfy the usual commutation relations and the modes $\phi_{0}$ and $\phi_{\nu}$ are normalized so that they satisfy

$$
\begin{aligned}
\left(\phi_{0} \cdot \phi_{0}\right) & =-\left(\phi_{0}^{*} \cdot \phi_{0}^{*}\right)=1, \\
\left(\phi_{\nu} \cdot \phi_{\nu^{\prime}}\right. & =-\left(\phi_{\nu}^{*} \cdot \phi_{\nu^{\prime}}^{*}\right)=\delta\left(\nu-\nu^{\prime}\right), \\
\left(\phi_{0} \cdot \phi_{\nu}\right) & =\left(\phi_{0}^{*} \cdot \phi_{\nu}^{*}\right)=0, \\
\left(\phi_{n} \cdot \phi_{n^{\prime}}^{*}\right) & =0, \quad \text { for } n, n^{\prime}=0, \nu,
\end{aligned}
$$


where $(\cdot)$ is the Wronskian defined by $[21,22]$

$$
(X \cdot Y):=-2 i \int_{\xi_{b}}^{\infty} d \xi \frac{\ell^{3}}{\eta^{2} \sinh ^{3} \xi}\left(X \partial_{\eta} Y^{*}-Y^{*} \partial_{\eta} X\right) .
$$

From the conditions (16) we can determine the normalization of the zero mode as

$$
\mathcal{N}=C(\ell H)
$$

with

$$
C(x):=\left[\sqrt{1+x^{2}}+x^{2} \ln \left(\frac{x}{1+\sqrt{1+x^{2}}}\right)\right]^{-1 / 2},
$$

which is the same as that introduced in Refs. [20-22], and behaves like $C^{2}(x) \approx 1$ for $x \ll 1$ and $C^{2}(x) \approx 3 x / 2$ for $x \gg 1$. This function plays an important role throughout the present paper.

The expectation value of the squared amplitude of the vacuum fluctuation in the zero mode is given by

$$
\left|\phi_{0}\right|^{2}=\ell^{-1} C^{2}(\ell H) \frac{H^{2}}{2 k^{3}}\left(1+k^{2} \eta^{2}\right) \rightarrow \ell^{-1} C^{2}(\ell H) \frac{H^{2}}{2 k^{3}},
$$

where the expression in the last expression is obtained by evaluating the perturbation in the superhorizon regime/at a late time, and hence this is the amplitude of the growing mode. In terms of the power spectrum defined by

$$
\mathcal{P}:=\frac{4 \pi k^{3}}{(2 \pi)^{3}} \cdot \frac{2}{\left(M_{5}\right)^{3}}\left|\phi_{0}\right|^{2},
$$

we have

$$
\mathcal{P}=\frac{2 C^{2}(\ell H)}{M_{\mathrm{Pl}}^{2}}\left(\frac{H}{2 \pi}\right)^{2},
$$

where we have used $\ell\left(M_{5}\right)^{3}=M_{\mathrm{Pl}}^{2}$. This is the standard flat spectrum up to the overall factor $C^{2}(\ell H)$. In conventional four-dimensional cosmology the power spectrum of the primordial gravitational waves from de Sitter inflation is given by [33]

$$
\mathcal{P}_{4 \mathrm{D}}=\frac{2}{M_{\mathrm{Pl}}^{2}}\left(\frac{H}{2 \pi}\right)^{2}
$$

Thus just by rescaling the amplitude in four-dimensional cosmology as

$$
H \mapsto H C(\ell H),
$$

the braneworld result (22) is exactly obtained.

\section{B. The junction model}

Pure de Sitter inflation on the brane described in the previous subsection is a special case where the amplitude of the growing zero mode can be obtained completely analytically. As a next step to understand gravitational waves from more general inflation with $H \neq$ const, a discontinuous change in the Hubble parameter was considered in Ref. [22]. In such a junction model the Hubble parameter is given by

$$
H(\eta)= \begin{cases}H_{i}, & \eta<\eta_{0} \\ H_{f}=H_{i}-\delta H, & \eta>\eta_{0}\end{cases}
$$

and

$$
\frac{\delta H}{H_{i}} \ll 1,
$$

is assumed.

The evolution of gravitational wave perturbations can be analyzed by solving the equation of motion backward using the advanced Green's function [21]. A set of mode functions can be constructed for the initial de Sitter stage, and another for the final de Sitter stage, either of which forms a complete orthonormal basis for the graviton wave function. The final (growing) zero mode may be written as a linear combination of the initial zero and KK modes. The Bogoliubov coefficients give the creation rate of final zero mode gravitons from the initial vacuum fluctuations in the Kaluza-Klein modes as well as in the zero mode. Thus the power spectrum may be written as a sum of two separate contributions,

$$
\mathcal{P}=\mathcal{P}_{0}+\mathcal{P}_{\mathrm{KK}},
$$

where $\mathcal{P}_{0}$ and $\mathcal{P}_{\mathrm{KK}}$ are the parts coming from the initial zero and Kaluza-Klein modes, respectively.

An important result brought by analyzing this junction model is that $\mathcal{P}$ is well approximated by the rescaled spectrum $\mathcal{P}_{\text {res }}$ obtained by using a simple map speculated by the exact result of pure de Sitter inflation [Eq. (24)]. More precisely, let $\mathcal{P}_{4 \mathrm{D}}(k)$ be the gravitational wave spectrum evaluated in the standard four-dimensional inflationary Universe with the same time dependence of the Hubble parameter (25), and then the rescaled spectrum is defined by

$$
\mathcal{P}_{4 \mathrm{D}}=\frac{2}{M_{\mathrm{Pl}}^{2}}\left(\frac{h_{k}}{2 \pi}\right)^{2} \mapsto \mathcal{P}_{\text {res }}=\frac{2 C^{2}\left(\ell h_{k}\right)}{M_{\mathrm{Pl}}^{2}}\left(\frac{h_{k}}{2 \pi}\right)^{2} .
$$

Comparing the rescaled spectrum with $\mathcal{P}$ obtained from a five-dimensional calculation, one finds that the difference between these two is suppressed to be second order like [22]

$$
\left|\frac{\mathcal{P}-\mathcal{P}_{\text {res }}}{\mathcal{P}}\right| \lesssim\left(\frac{\delta H}{H_{i}}\right)^{2} \ll 1 .
$$

It might be worth noting here that the KK contribution $\mathcal{P}_{\mathrm{KK}}$ is necessary for realizing this interesting agreement between the braneworld result and the (basically) fourdimensional result especially at high energies $\ell H \gg 1$. The above agreement (29) raised a speculation that the mapping formula $h_{k} \mapsto C\left(\ell h_{k}\right)$ may work with good accu- 
racy in more general inflation models with a smoothly changing expansion rate. The central purpose of the present paper is to check whether this speculation is correct or not.

\section{FORMULATION}

\section{A. Basic equations}

Now let us explain the formulation that we use to study the generation of gravitational waves without assuming a pure de Sitter brane. Our formulation is based on double null coordinates, which are presumably the most convenient for numerical calculations.

The metric (1) can be rewritten by using double null coordinates

$$
\begin{aligned}
& u=t-z, \\
& v=t+z
\end{aligned}
$$

in the form of

$$
d s^{2}=\frac{4 \ell^{2}}{(v-u)^{2}}\left(-d u d v+\delta_{i j} d x^{i} d x^{j}\right) .
$$

The trajectory of the brane can be specified arbitrarily by

$$
v=q(u) \text {. }
$$

By a further coordinate transformation

$$
\begin{gathered}
U=u, \\
q(V)=v,
\end{gathered}
$$

we obtain

$$
d s^{2}=\frac{4 \ell^{2}}{[q(V)-U]^{2}}\left[-q^{\prime}(V) d U d V+\delta_{i j} d x^{i} d x^{j}\right],
$$

where a prime denotes differentiation with respect to the argument. Now in the new coordinates the position of the brane is simply given by

$$
U=V \text {. }
$$

We will use this coordinate system for actual numerical calculations.

The induced metric on the brane is

$$
d s_{b}^{2}=\frac{4 \ell^{2}}{[q(V)-V]^{2}}\left[-q^{\prime}(V) d V^{2}+\delta_{i j} d x^{i} d x^{j}\right],
$$

from which we can read off the conformal time $\eta$ and the scale factor $a$, respectively, as

$$
\begin{gathered}
d \eta=\sqrt{q^{\prime}(V)} d V, \\
a=\frac{2 \ell}{q(V)-V},
\end{gathered}
$$

and hence the Hubble parameter on the brane is written as

$$
\ell H=\frac{1}{2 \sqrt{q^{\prime}(V)}}\left[1-q^{\prime}(V)\right]
$$

or equivalently

$$
q^{\prime}(V)=\left(\sqrt{1+\ell^{2} H^{2}}-\ell H\right)^{2} .
$$

Given the Hubble parameter as a function of $\eta$, one can integrate Eqs. (39) and (42) to obtain $q$ as a function of $V$.

When the brane undergoes pure de Sitter inflation [and thus $q^{\prime}(V)=$ constant], the following relation between $(U, V)$ and $(\eta, \xi)$ will be useful:

$$
\begin{gathered}
\xi=\xi_{b}+\frac{1}{2} \ln \left(\frac{t_{0}-U}{t_{0}-V}\right), \\
\eta=-e^{-\xi_{b}}\left[\left(t_{0}-U\right)\left(t_{0}-V\right)\right]^{1 / 2},
\end{gathered}
$$

and

$$
q(V)=e^{-2 \xi_{b}}\left(V-t_{0}\right)+t_{0} .
$$

The Klein-Gordon-type equation for a gravitational wave perturbation $\phi$ in the $(U, V)$ coordinates reduces to $\left[4 \partial_{U} \partial_{V}+\frac{6}{q(V)-U}\left(\partial_{V}-q^{\prime}(V) \partial_{U}\right)+q^{\prime}(V) k^{2}\right] \phi=0$,

supplemented by the boundary condition

$$
\left.\left[\partial_{U}-\partial_{V}\right] \phi\right|_{U=V}=0 .
$$

The expression for the Wronskian evaluated on a constant $V$ hypersurface is given by

$$
(X \cdot Y)=2 i \int_{-\infty}^{V} d U\left[\frac{2 \ell}{q(V)-U}\right]^{3}\left(X \partial_{U} Y^{*}-Y^{*} \partial_{U} X\right),
$$

which is independent of the choice of the hypersurface.

When inflation on the brane deviates from a pure de Sitter one, the decomposition into the zero mode and KK modes becomes rather ambiguous. For this reason we require the initial and final phases of inflation to be pure de Sitter, though arbitrary cosmic expansion is allowed in the intermediate stage. In both de Sitter phases, $q(V)$ can be fit by Eq. (45), and $\xi_{b}$ and $t_{0}$ are determined, respectively. Hence we have two sets of de Sitter coordinates $(\eta, \xi)$ and $(\tilde{\eta}, \tilde{\xi})$. We distinguish the coordinates in the final phase by associating them with tilde. In the final de Sitter phase the mode will be well outside the horizon, and hence we expand the graviton field in terms of the growing and decaying zero mode solutions $\tilde{\phi}_{g}$ and $\tilde{\phi}_{d}$ as

$$
\phi=\hat{A}_{g} \tilde{\phi}_{g}+\hat{A}_{d} \tilde{\phi}_{d}+\int d \nu\left(\hat{A}_{\nu} \tilde{\phi}_{\nu}+\hat{A}_{\nu}^{\dagger} \tilde{\phi}_{\nu}^{*}\right),
$$

where

$$
\tilde{\phi}_{g}:=\operatorname{Im}\left[\tilde{\phi}_{0}\right],
$$




$$
\tilde{\phi}_{d}:=\operatorname{Re}\left[\tilde{\phi}_{0}\right],
$$

and the mode functions with tildes are defined in the same way as $\phi_{0}$ and $\phi_{\nu}$ with the substitution of $(\tilde{\xi}, \tilde{\eta})$ for $(\xi, \eta)$. It can be easily seen that the growing and the decaying modes are normalized as

$$
\left(\tilde{\phi}_{g} \cdot \tilde{\phi}_{d}\right)_{f}=\frac{1}{2}, \quad\left(\tilde{\phi}_{g} \cdot \tilde{\phi}_{g}\right)_{f}=\left(\tilde{\phi}_{d} \cdot \tilde{\phi}_{d}\right)_{f}=0,
$$

where subscript $f$ means that the expression is to be evaluated in the final de Sitter phase. Notice that de Sitter mode functions thus defined as functions of $(U, V)$ through $(\xi, \eta)$ [or $(\tilde{\xi}, \tilde{\eta})]$ do not satisfy the equation of motion outside the initial (or final) de Sitter phase. Back in the initial de Sitter phase the graviton field can be expanded as

$$
\phi=\hat{a}_{0} \phi_{0}+\hat{a}_{0}^{\dagger} \phi_{0}^{*}+\int d \nu\left(\hat{a}_{\nu} \phi_{\nu}+\hat{a}_{\nu}^{\dagger} \phi_{\nu}^{*}\right)
$$

We assume that initially the gravitons are in the de Sitter invariant vacuum state annihilated by $\hat{a}_{0}$ and $\hat{a}_{\nu}$,

$$
\hat{a}_{0}|0\rangle=\hat{a}_{\nu}|0\rangle=0 \text {. }
$$

We would like to evaluate the expectation value of the squared amplitude of the vacuum fluctuation in the growing mode at a late time,

$$
\left|\tilde{\phi}_{g}\right|^{2}\left\langle 0\left|\hat{A}_{g}^{2}\right| 0\right\rangle,
$$

or equivalently, the power spectrum,

$$
\begin{aligned}
\mathcal{P}(k) & =\frac{4 \pi k^{3}}{(2 \pi)^{3}} \frac{2}{\left(M_{5}\right)^{3}} \cdot\left|\tilde{\phi}_{g}\right|^{2}\left\langle 0\left|\hat{A}_{g}^{2}\right| 0\right\rangle \\
& \rightarrow \frac{2 C^{2}\left(\ell H_{f}\right)}{M_{\mathrm{Pl}}^{2}}\left(\frac{H_{f}}{2 \pi}\right)^{2}\left\langle 0\left|\hat{A}_{g}^{2}\right| 0\right\rangle,
\end{aligned}
$$

where $H_{f}$ is the Hubble parameter in the final de Sitter phase. In order to obtain the final amplitude, it is not necessary to solve the evolution of all the (infinite number of) degrees of freedom with their initial conditions set by Eq. (53). In fact, we have only to solve the backward evolution of the final decaying mode, as explained below.

Suppose that a solution $\Phi(U, V)$ is chosen so as to satisfy $\Phi=\tilde{\phi}_{d}$ in the final de Sitter phase. From the Wronskian condition (52) we see that

$$
\begin{aligned}
\frac{1}{2} \hat{A}_{g} & =\left(\phi \cdot \tilde{\phi}_{d}\right)_{f}=(\phi \cdot \Phi) \\
& =\left(\phi_{0} \cdot \Phi\right)_{i} \hat{a}_{0}+\int d \nu\left(\phi_{\nu} \cdot \Phi\right)_{i} \hat{a}_{\nu}+\text { h.c. }
\end{aligned}
$$

where subscript $i$ means that the expression is to be evaluated in the initial de Sitter phase. In the above we used the fact that the Wronskian is constant in time. Thus we obtain

$$
\left\langle 0\left|\hat{A}_{g}^{2}\right| 0\right\rangle=4\left[\left|\left(\phi_{0} \cdot \Phi\right)_{i}\right|^{2}+\int d \nu\left|\left(\phi_{\nu} \cdot \Phi\right)_{i}\right|^{2}\right],
$$

from which we can calculate the power spectrum of the primordial gravitational waves. It is obvious that the spectrum is written in the form of Eq. (27) with

$$
\begin{gathered}
\mathcal{P}_{0}:=\frac{8 C^{2}\left(\ell H_{f}\right)}{M_{\mathrm{Pl}}^{2}}\left(\frac{H_{f}}{2 \pi}\right)^{2}\left|\left(\phi_{0} \cdot \Phi\right)_{i}\right|^{2}, \\
\mathcal{P}_{\mathrm{KK}}:=\frac{8 C^{2}\left(\ell H_{f}\right)}{M_{\mathrm{Pl}}^{2}}\left(\frac{H_{f}}{2 \pi}\right)^{2} \int d \nu\left|\left(\phi_{\nu} \cdot \Phi\right)_{i}\right|^{2} .
\end{gathered}
$$

\section{B. Numerical scheme}

Here we describe the algorithm that we employ to solve the backward evolution of the gravitational perturbations numerically. We set the boundary conditions for the mode $\Phi$ so as to be identical to a decaying zero mode $\tilde{\phi}_{d}$ at a time $V=V_{f}$ in the final de Sitter phase. We decompose $\Phi$ as

$$
\Phi(U, V)=\tilde{\phi}_{d}(U, V)+\delta \Phi(U, V),
$$

and solve the equation for $\delta \Phi(U, V)$ instead of $\Phi(U, V)$. The equation of motion for $\delta \Phi$ is obtained as

$$
\begin{aligned}
& {\left[4 \partial_{U} \partial_{V}+\frac{6}{q(V)-U}\left(\partial_{V}-q^{\prime}(V) \partial_{U}\right)+q^{\prime}(V) k^{2}\right] \delta \Phi} \\
& =-\left[4 \partial_{U} \partial_{V}+\frac{6}{q(V)-U}\left(\partial_{V}-q^{\prime}(V) \partial_{U}\right)+q^{\prime}(V) k^{2}\right] \tilde{\phi}_{d}
\end{aligned}
$$

Since both $\Phi$ and $\tilde{\phi}_{d}$ satisfy the boundary condition of the form of Eq. (47), the boundary condition for $\delta \Phi$ is also written as

$$
\left.\left[\partial_{U}-\partial_{V}\right] \delta \Phi\right|_{U=V}=0 .
$$

We immediately find that we do not have to solve the backward evolution in the final de Sitter phase, since $\delta \Phi$ identically vanishes there.

In some cases $\Phi$ is not disturbed so much from its final configuration $\tilde{\phi}_{d}\left(U, V_{f}\right)$, especially when we discuss a small deviation from the pure de Sitter case. It is advantageous then to use a small quantity $\delta \Phi$ as a variable in numerical calculations. Of course, there is no problem even when $\delta \Phi$ does not stay small.

Our scheme to obtain the values of $\delta \Phi$ at the initial surface $V=V_{i}$ is as follows. We give the boundary conditions at $V=V_{f}$ as $f_{1,1}, f_{2,1}, \ldots, f_{N, 1}=0$, where

$$
f_{n, m}:=\delta \Phi\left(U=V_{f}-\varepsilon(n-1), V=V_{f}-\varepsilon(m-1)\right) .
$$

A sketch of the numerical grids is shown in Fig. 1. Here $N$ is taken to be sufficiently large, and we use the same grid spacing $\varepsilon$ both in the $U$ and $V$ directions. At a virtual site $(U, V)=\left(V_{f}, V_{f}-\varepsilon\right)$ we set 
TSUTOMU KOBAYASHI AND TAKAHIRO TANAKA

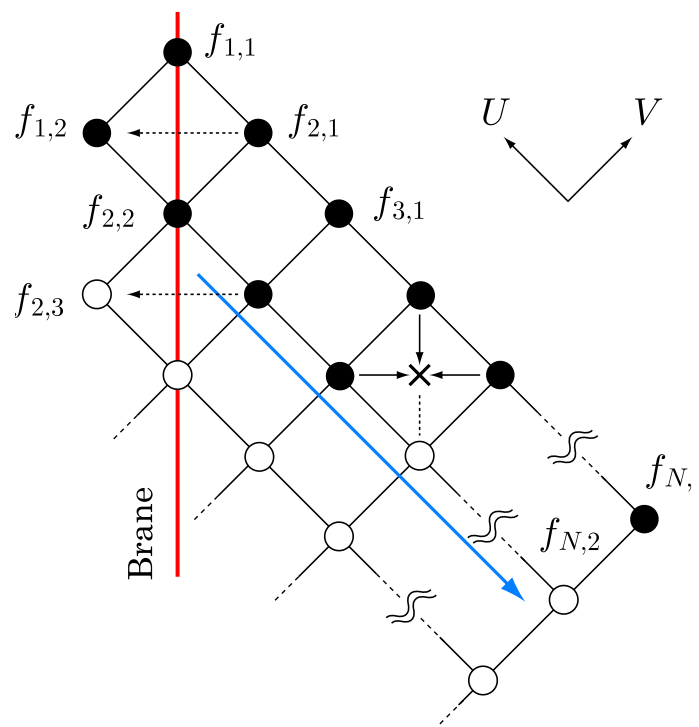

FIG. 1 (color online). Numerical (backward) evolution scheme.

$$
f_{1,2}=f_{2,1} \text {, }
$$

so that the boundary condition (61) is satisfied at $\left(V_{f}-\right.$ $\left.\varepsilon / 2, V_{f}-\varepsilon / 2\right)$. From $f_{1,1}, f_{2,1}$, and $f_{1,2}$ we can determine the value of $f_{2,2}$ by using the equation of motion (60) at $\left(V_{f}-\varepsilon / 2, V_{f}-\varepsilon / 2\right)$, and then from $f_{2,1}, f_{3,1}$, and $f_{2,2}$ we can determine $f_{3,2}$, and so on. We repeat the same procedure in the subsequent time steps until we obtain $f_{M, M}, f_{M+1, M}, \ldots, f_{N, M}$ at $V_{i}=V_{f}-\varepsilon(M-1)$.

\section{A toy model}

As stated above, to make the problem well posed, we consider models which have initial and final de Sitter phases. To perform numerical calculations, as a concrete example, we adopt a simple toy model in which the Hubble parameter is given by an analytic form

$$
H(\eta)=\bar{H}-\Delta \tanh \left(\frac{\eta-\eta_{0}}{s}\right),
$$

where

$$
\bar{H}:=\frac{H_{i}+H_{f}}{2}, \quad \Delta:=\frac{H_{i}-H_{f}}{2},
$$

with $H_{i}$ and $H_{f}$ the initial and final values of the Hubble parameter; $s$ is a parameter that controls the smoothness of the transition, and $\eta_{0}$ indicates a transition time. Taking the initial time $\eta_{i}$ and the final time $\eta_{f}$ so that $\eta_{0}-\eta_{i} \gg s$ and $\eta_{f}-\eta_{0} \approx-\eta_{0} \gg s$, we have $H \approx H_{i}$ for $\eta \rightarrow \eta_{i}$ and $H \approx H_{f}$ for $\eta \rightarrow \eta_{f}$. The scale factor for this inflation model is given by

$$
a(\eta)=\left\{s \Delta \ln \left[2 \cosh \left(\frac{\eta-\eta_{0}}{s}\right)\right]-\bar{H} \eta+\Delta \eta_{0}\right\}^{-1}
$$

PHYSICAL REVIEW D 71, 124028 (2005)
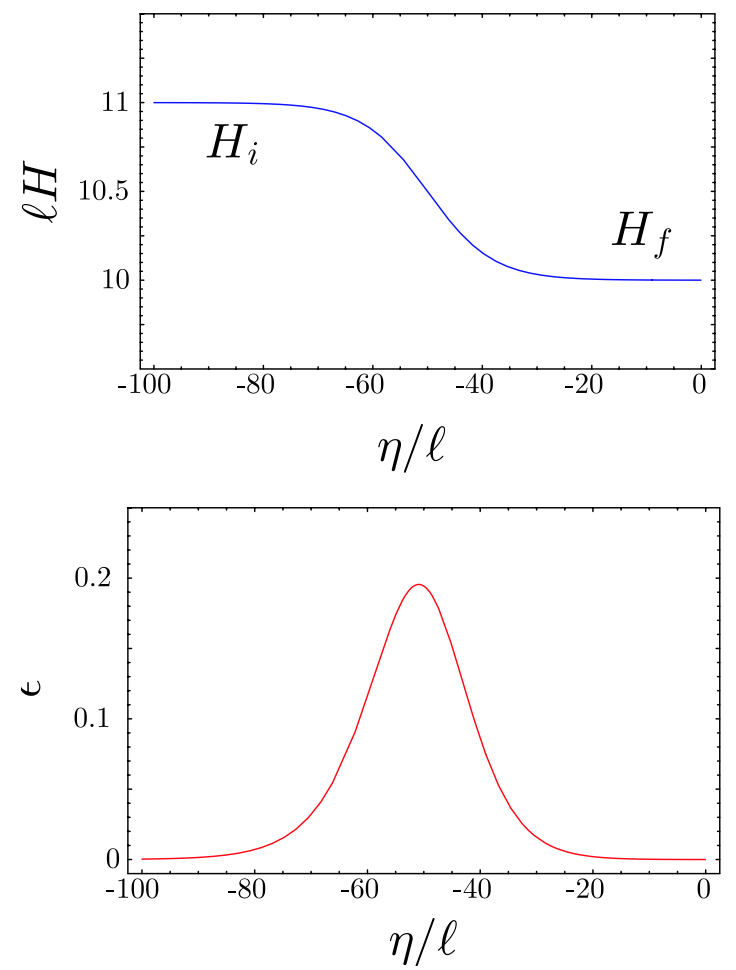

FIG. 2 (color online). The Hubble parameter and the slow-roll parameter $\epsilon$ in our model. This is a plot for the model of Fig. 9.

where the integration constant is determined by imposing that $a \approx\left(-H_{f} \eta\right)^{-1}$ at $\eta \approx \eta_{f} \approx 0$. The slow-roll parameter, $\epsilon:=-\partial_{\eta} H /\left(a H^{2}\right)$, is obtained as

$$
\epsilon=\frac{\Delta\left\{s \Delta \ln \left[2 \cosh \left(\left(\eta-\eta_{0}\right) / s\right)\right]-\bar{H} \eta+\Delta \eta_{0}\right\}}{s\left[\bar{H} \cosh \left(\left(\eta-\eta_{0}\right) / s\right)-\Delta \sinh \left(\left(\eta-\eta_{0}\right) / s\right)\right]^{2}},
$$

which takes maximum at $\eta=\eta_{0}$. The maximum value is

$$
\epsilon\left(\eta_{0}\right)=\epsilon_{\max }=\frac{\Delta}{s \bar{H}^{2} a\left(\eta_{0}\right)} .
$$

The behavior of the Hubble parameter and the slow-roll parameter is shown in Fig. 2.

Here we should mention the limitation of this simple toy model. For fixed values of $H_{i}, H_{f}$, and $\epsilon_{\max }$ one can prolong the period of the transition $\sim s$ measured in conformal time by taking large $s$. However, using the definition of $\epsilon_{\max }(67)$, the transition time scale in proper time is found to be given by $\sim a\left(\eta_{0}\right) s=\bar{H}^{2} \epsilon_{\max } / \Delta$. Therefore we cannot change the transition time scale independently of the other parameters, $H_{i}, H_{f}$, and $\epsilon_{\max }$.

\section{NUMERICAL RESULTS}

Using various parameters shown in Table I we performed numerical calculations, the results of which are presented in Figs. 3-10. Numbers of grids are $N \times M=$ $50000 \times 1000$, and the grid separation $\varepsilon$ is chosen to be 


\section{QUANTUM-MECHANICAL GENERATION OF ...}

TABLE I. Parameters used for the numerical calculations presented in the figures.

\begin{tabular}{l|ccc|cc}
\hline \hline & $\ell H_{i}$ & $\ell H_{f}$ & $\epsilon_{\max }$ & $\ell / s$ & $\eta_{0} / \ell$ \\
\hline Figure 3 & 0.11 & 0.10 & 0.21 & 0.09 & -50.45 \\
Figure 4 & 0.11 & 0.08 & 0.69 & 0.1 & -50.46 \\
Figure 5 & 1.1 & 1.0 & 0.21 & 0.09 & -50.21 \\
Figure 6 & 1.25 & 1.0 & 0.45 & 0.09 & -50.21 \\
Figure 7 & 1.5 & 1.0 & 0.75 & 0.09 & -50.21 \\
Figure 8 & 3.5 & 3.0 & 0.34 & 0.095 & -50.08 \\
Figure 9 & 11 & 10 & 0.19 & 0.085 & -50.02 \\
Figure 10 & 15 & 10 & 0.75 & 0.09 & -50.02 \\
\hline \hline
\end{tabular}

(0.11-2.5) $\times \ell$ depending on the energy scale of inflation. Note here that the step width in conformal time $\eta$ is given by $\Delta \eta=\sqrt{q^{\prime}(V)} \varepsilon$ [Eq. (39)], and $q^{\prime}(V)$ becomes smaller for a larger value of $\ell H$ [Eq. (42)]. Our choice of $\varepsilon$ makes $\Delta \eta$ about the same size, $\Delta \eta \approx 0.1$, in all the calculations. Integration over $\nu$ is performed up to $\nu=20-40$ with an
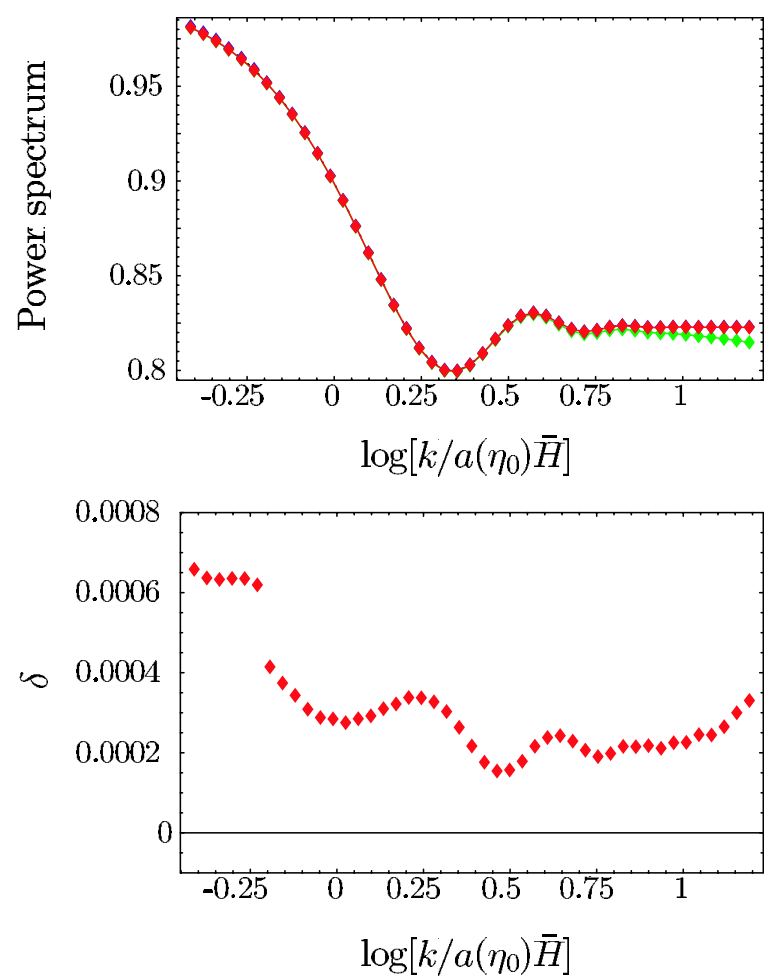

FIG. 3 (color online). Top panel: power spectra of gravitational waves normalized by $\left(2 C^{2}\left(\ell H_{i}\right) / M_{\mathrm{PI}}^{2}\right)\left(H_{i} / 2 \pi\right)^{2}$. Red diamonds (upper ones) indicate the result including the contributions from the initial Kaluza-Klein modes, while green diamonds (lower ones) represent the contribution from the initial zero mode. Although the rescaled four-dimensional spectrum is shown by blue diamonds, they are almost hidden by the red ones since the result of the five-dimensional calculation is well approximated by the rescaled four-dimensional spectrum. Bottom panel: difference between the five-dimensional and rescaled four-dimensional spectra.
PHYSICAL REVIEW D 71, 124028 (2005)
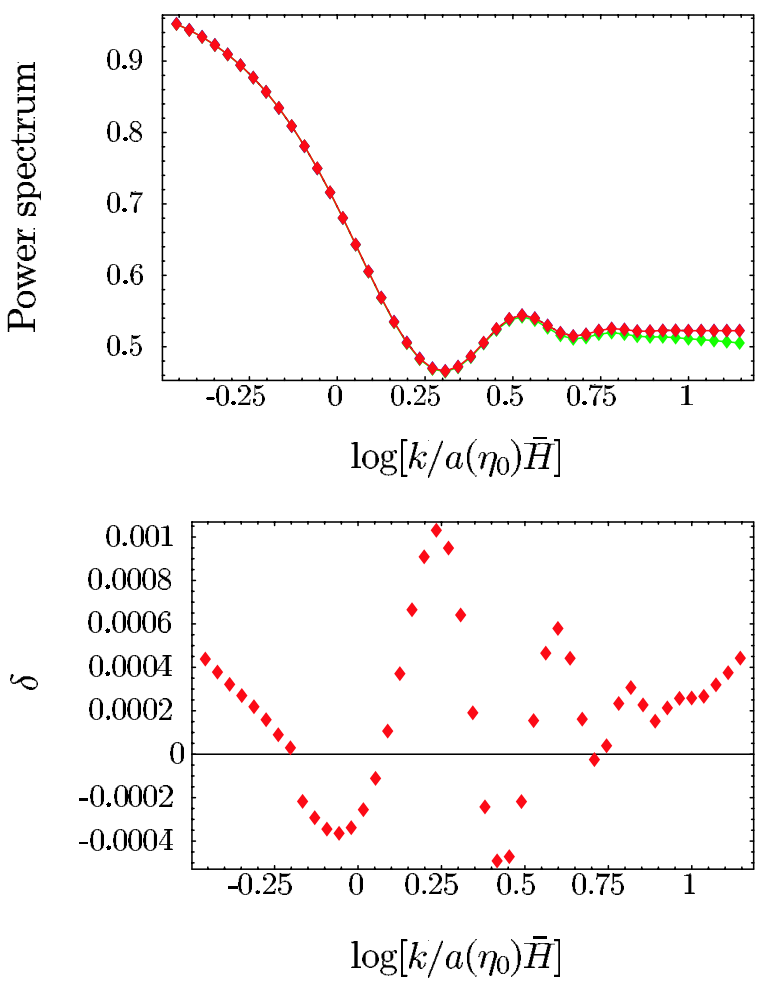

FIG. 4 (color online). Same as Fig. 3, but the parameters are different.
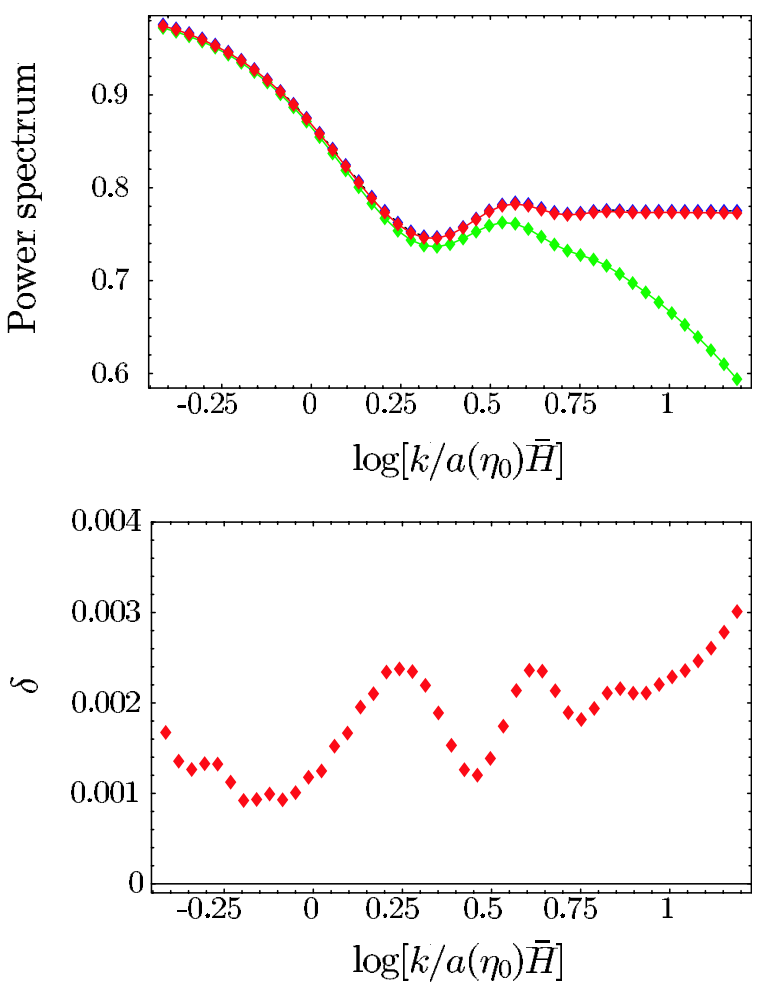

FIG. 5 (color online). Same as Fig. 3, but the parameters are different. 


\section{TSUTOMU KOBAYASHI AND TAKAHIRO TANAKA}
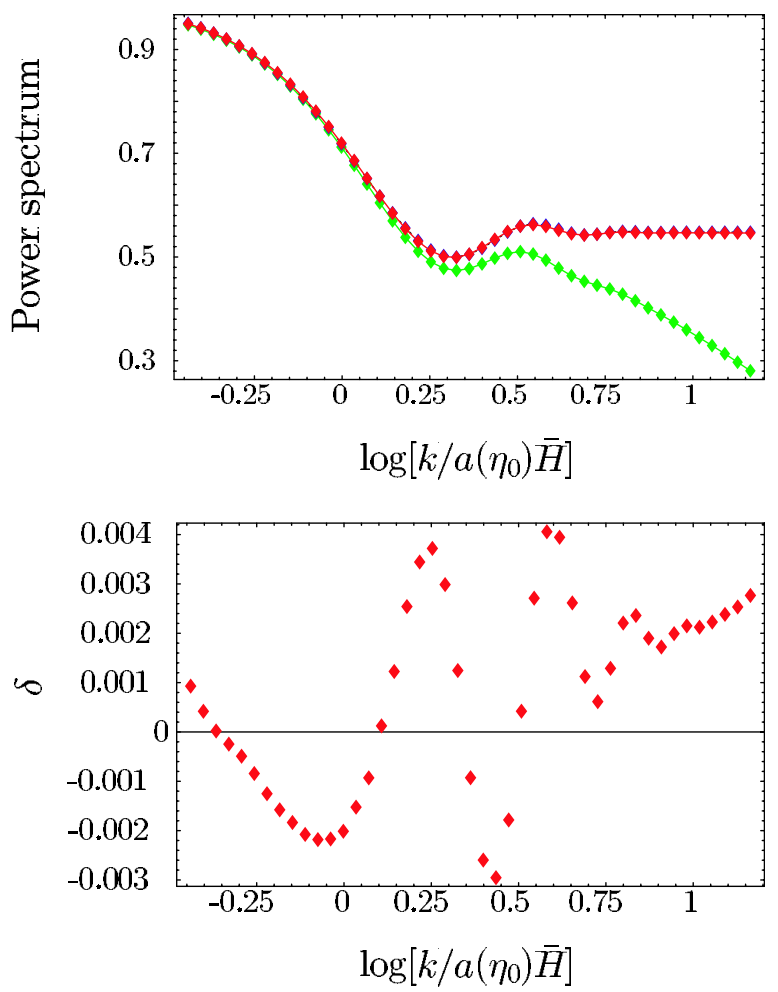

FIG. 6 (color online). Same as Fig. 3, but the parameters are different.
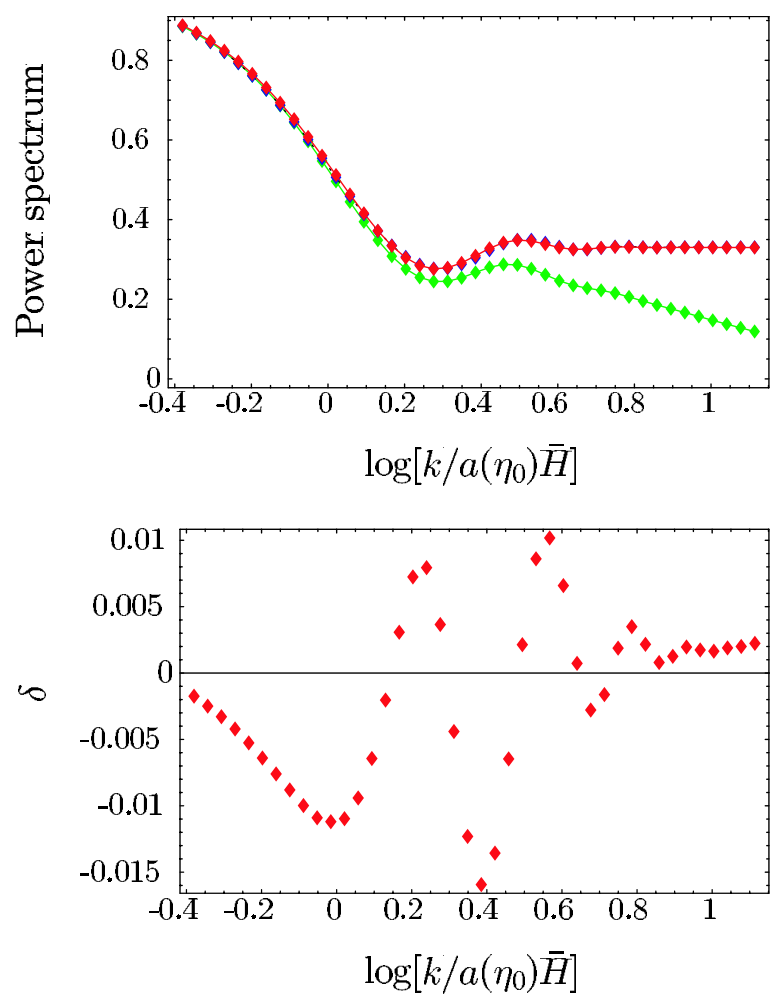

FIG. 7 (color online). Same as Fig. 3, but the parameters are different.
PHYSICAL REVIEW D 71, 124028 (2005)
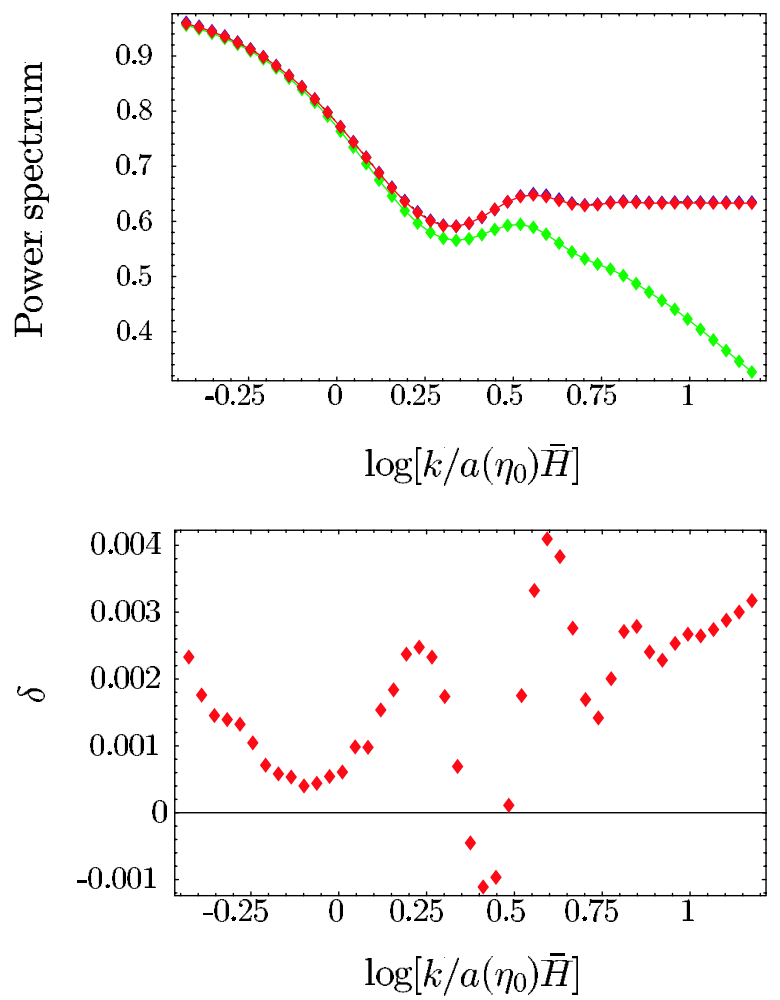

FIG. 8 (color online). Same as Fig. 3, but the parameters are different.
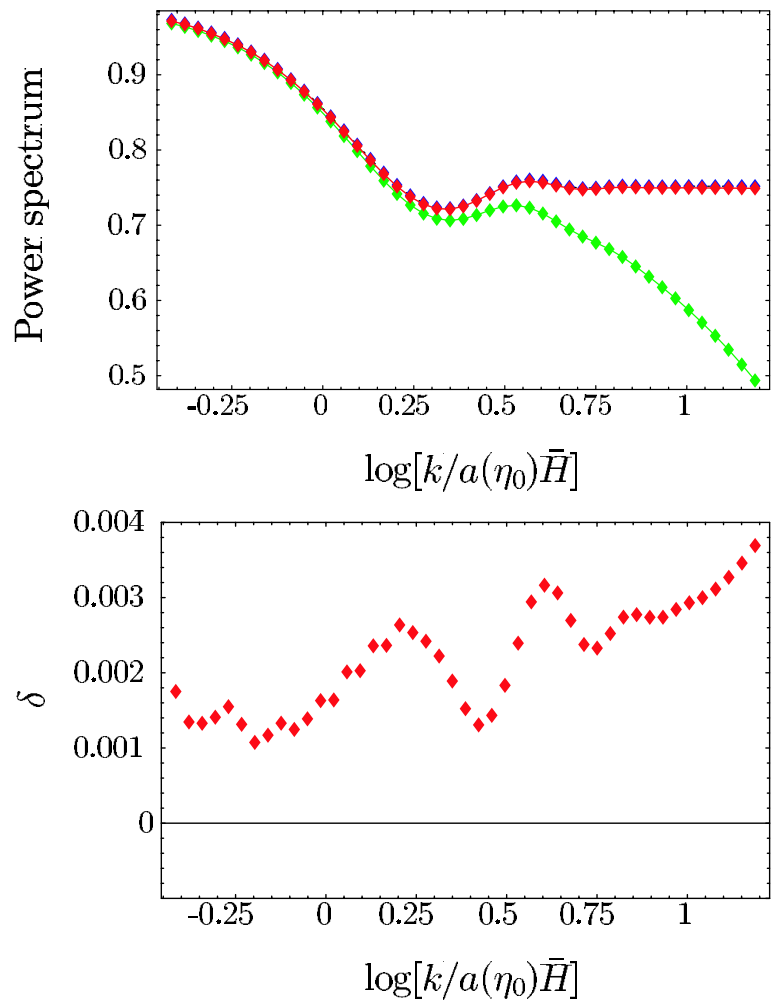

FIG. 9 (color online). Same as Fig. 3, but the parameters are different. 

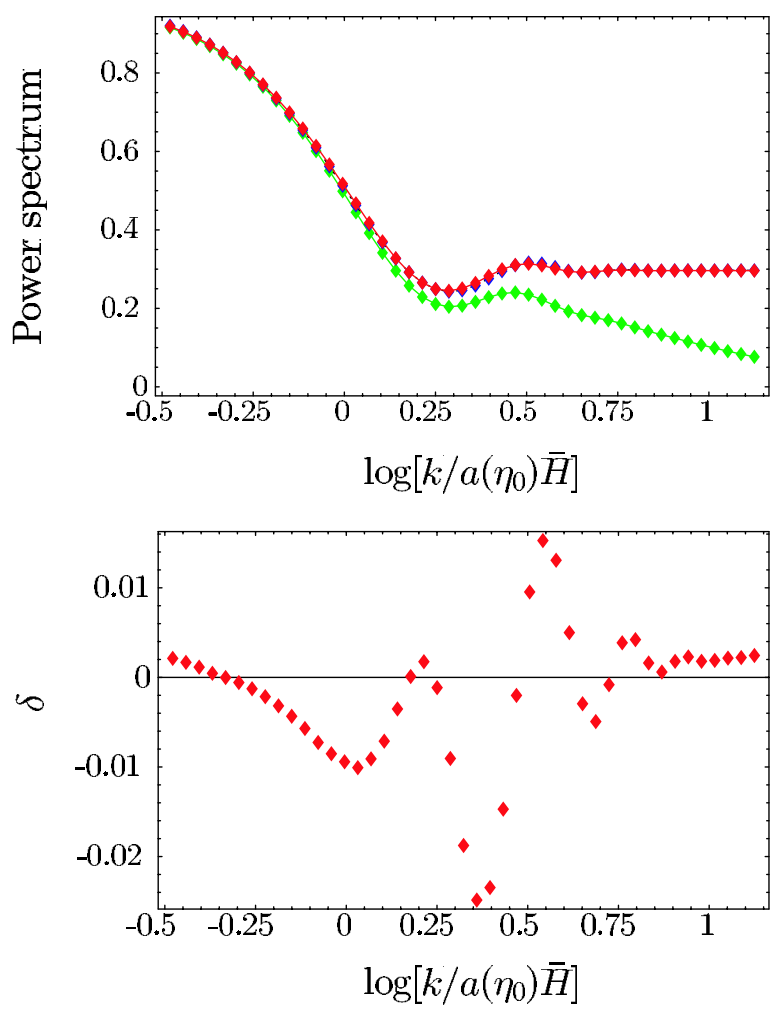

FIG. 10 (color online). Same as Fig. 3, but the parameters are different.

equal grid spacing of 0.15 . The typical behavior of the integrand $\left|\left(\phi_{\nu} \cdot \Phi\right)_{i}\right|^{2}$ is shown in Fig. 11.

In order to compare the five-dimensional power spectrum with a four-dimensional counterpart, we solve the conventional evolution equation for gravitational waves in four dimensions,

$$
\left(\partial_{\eta}^{2}+2 a H \partial_{\eta}+k^{2}\right) \Phi=0,
$$

where $H(\eta)$ is given by the same function as used for the corresponding five-dimensional computation, and then we calculate the rescaled power spectrum obtained from the four-dimensional "bare" spectrum by using the mapping formula (28).

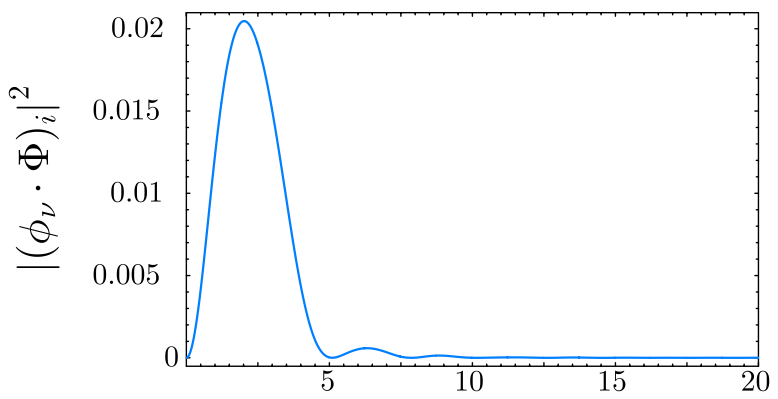

FIG. 11 (color online). Wronskian $\left|\left(\phi_{\nu} \cdot \Phi\right)_{i}\right|^{2}$ as a function of $\nu$. The parameters are given by those of Fig. 9 and $k / a\left(\eta_{0}\right) \bar{H}=10$.
We introduce a parameter that represents a difference between the five-dimensional power spectrum and the rescaled four-dimensional spectrum:

$$
\delta(k):=\frac{\mathcal{P}_{\text {res }}-\mathcal{P}}{\mathcal{P}} .
$$

In all the cases of our calculations we clearly see that $|\delta| \ll 1$ and thus we conclude that the primordial spectrum of the gravitational waves in the braneworld is quite well approximated by the rescaled four-dimensional spectrum. We also find that the difference becomes larger for larger values of the slow-roll parameter $\epsilon$, but $|\delta|$ is no greater than $\mathcal{O}\left(10^{-2}\right)$ even for models with $\epsilon_{\max } \simeq 0.75$. Notice that the Universe is not inflating any more if $\epsilon$ is greater than unity. At high energies $\ell H \gtrsim 1$ it can be seen that dependence of $\delta$ on $\ell H$ is weak, while at low energies like $\ell H \sim 0.1, \delta$ is further suppressed compared to the high-energy cases.

Contributions from the initial KK modes can be significant for small wavelength modes. For example, for the modes with $k / a\left(\eta_{0}\right) \bar{H} \simeq 10$ we see that $\mathcal{P}_{\mathrm{KK}} / \mathcal{P} \sim$ $\mathcal{O}\left(10^{-2}\right)$ at low energies, but the KK contribution can become as large as $\mathcal{P}_{\mathrm{KK}} / \mathcal{P} \sim 0.6$ in our most "violent" model with $\ell H \sim 10$ and $\epsilon_{\max } \simeq 0.75$.

\section{DISCUSSION}

In this paper we have investigated the generation of gravitational waves during inflation on a brane and computed the primordial spectrum. Extending the previous work [22], we have considered a model composed of the initial and final de Sitter stages, and the transition region connecting them smoothly. We have numerically solved the backward evolution of the final decaying mode to obtain the amplitude of the growing zero mode at a late time by making use of the Wronskian method.

We found that the power spectrum $\mathcal{P}$ is well approximated by the rescaled spectrum $\mathcal{P}_{\text {res }}$ basically calculated in standard four-dimensional inflationary cosmology. Here the rescaling formula for the amplitude is given by a simple map $h_{k} \mapsto h_{k} C\left(\ell h_{k}\right)$, where $C$ is the normalization factor of the zero mode. Although the difference of the two spectra, $\delta:=\left(\mathcal{P}_{\text {res }}-\mathcal{P}\right) / \mathcal{P}$, depends on the energy scale of inflation and the slow-roll parameter, our numerical analysis clearly shows that in any case the mapping formula works with quite good accuracy, yielding $|\delta| \lesssim$ $\mathcal{O}\left(10^{-2}\right)$. This implies that the mapping relation between the two spectra holds quite generally in the RandallSundrum braneworld, which we believe is a useful formula.

We have taken into account the vacuum fluctuations in initial Kaluza-Klein modes as well as the zero mode, and found that both of them contribute to the final amplitude of the zero mode. The amount of the initial KK contribution can be large on small scales, and it increases as the energy scale of inflation $\ell H$ becomes higher. This gives rise to a 
quite interesting picture. When the expansion rate changes during inflation, zero mode gravitons escape into the bulk as KK gravitons, but at the same time bulk gravitons come onto the brane to compensate for the loss, and these two effects almost cancel each other. This seems to happen, irrespective of the energy scale, in a wide class of the inflation models even with a not-so-small slow-roll parameter. It is suggested that this is not the case in the radiation dominated decelerating Universe [23-26], where the decay into KK gravitons reduces the amplitude of the gravitational waves on the brane. The junction model of Ref. [21] joining de Sitter and Minkowski branes also shows the suppression of the gravitational wave amplitude at high energies.

What is the reason for the remarkable agreement of the braneworld spectrum and the rescaled four-dimensional spectrum? Extremely long wavelength modes, which leave the horizon during the initial de Sitter stage much before the Hubble parameter changes, have a squared amplitude of $\left(2 C^{2}\left(\ell H_{i}\right) / M_{\mathrm{Pl}}^{2}\right)\left(H_{i} / 2 \pi\right)^{2}$, and the amplitude of the perturbations stays constant during the subsequent stages [34]. The same is true for four-dimensional inflationary cosmology, and so the mapping formula is applicable to these long wavelength modes. On the other hand, a mode whose wavelength is much shorter than the Hubble horizon scale at the transition time will feel the transition as adiabatic and hence the particle production is exponentially suppressed, $\left\langle 0\left|\hat{A}_{g}^{\dagger} \hat{A}_{g}\right| 0\right\rangle \approx 1$, leading to $\mathcal{P} \approx$ $\left(2 C^{2}\left(\ell H_{f}\right) / M_{\mathrm{Pl}}^{2}\right)\left(H_{f} / 2 \pi\right)^{2}$. The same argument can be applied to the conventional cosmology. Thus it is not surprising that the mapping formula works for such short wavelength modes. However, at present there seems no simple reason for the amplitude of the modes with $k \sim$ $\mathcal{O}\left(a\left(\eta_{0}\right) \bar{H}\right)$ to coincide with the rescaled one.

\section{ACKNOWLEDGMENTS}

We thank Shinji Mukohyama for valuable discussions. This work was supported in part by the JSPS, by Grant-inAid for Scientific Research, No. 14047212 and No. 16740141, and by the Grant-in-Aid for the 21st
Century COE "Center for Diversity and Universality in Physics" from the Ministry of Education, Culture, Sports, Science and Technology of Japan.

\section{APPENDIX: KALUZA-KLEIN MODE FUNCTIONS}

In the de Sitter braneworld the Kaluza-Klein mode functions in the time direction satisfy Eq. (12) and are given by

$$
\psi_{\nu}(\eta)=\frac{\sqrt{\pi}}{2} \ell^{-3 / 2} e^{-\pi \nu / 2}(-\eta)^{3 / 2} H_{i \nu}^{(1)}(-k \eta),
$$

where $H_{i \nu}^{(1)}$ is the Hankel function. They are normalized so that

$$
-\frac{i \ell^{3}}{\eta^{2}}\left(\psi_{\nu} \partial_{\eta} \psi_{\nu}^{*}-\psi_{\nu}^{*} \partial_{\eta} \psi_{\nu}\right)=1 .
$$

The mode functions in the extra direction satisfy Eq. (13) and are given by

$$
\begin{aligned}
\chi_{\nu}(\xi)= & C_{1}(\sinh \xi)^{2}\left[P_{-1 / 2+i \nu}^{-2}(\cosh \xi)\right. \\
& \left.-C_{2} Q_{-1 / 2+i \nu}^{-2}(\cosh \xi)\right]
\end{aligned}
$$

where $P_{-1 / 2+i \nu}^{-2}$ and $Q_{-1 / 2+i \nu}^{-2}$ are the associated Legendre functions. The constants $C_{1}$ and $C_{2}$ are determined by the normalization condition

$$
2 \int_{\xi_{b}}^{\infty} \frac{d \xi}{(\sinh \xi)^{3}} \chi_{\nu^{\prime}}^{*} \chi_{\nu}=\delta\left(\nu-\nu^{\prime}\right)
$$

and the boundary condition (47), respectively, as

$$
\begin{gathered}
C_{1}=\left[\left|\frac{\Gamma(i \nu)}{\Gamma(5 / 2+i \nu)}\right|^{2}+\mid \frac{\Gamma(-i \nu)}{\Gamma(5 / 2-i \nu)}\right. \\
\left.-\left.\pi C_{2} \frac{\Gamma(i \nu-3 / 2)}{\Gamma(1+i \nu)}\right|^{2}\right]^{-1 / 2} \\
C_{2}=\frac{P_{-1 / 2+i \nu}^{-1}\left(\cosh \xi_{b}\right)}{Q_{-1 / 2+i \nu}^{-1}\left(\cosh \xi_{b}\right)}
\end{gathered}
$$

[1] For a nice review, see, e.g., R. Maartens, Living Rev. Relativity 7, 7 (2004).

[2] L. Randall and R. Sundrum, Phys. Rev. Lett. 83, 4690 (1999).

[3] J. Garriga and T. Tanaka, Phys. Rev. Lett. 84, 2778 (2000); S. B. Giddings, E. Katz, and L. Randall, J. High Energy Phys. 03 (2000) 023.

[4] T. Shiromizu, K. i. Maeda, and M. Sasaki, Phys. Rev. D 62, 024012 (2000).

[5] P. Binetruy, C. Deffayet, and D. Langlois, Nucl. Phys.
B565, 269 (2000); P. Binetruy, C. Deffayet, U. Ellwanger, and D. Langlois, Phys. Lett. B 477, 285 (2000); S. Mukohyama, Phys. Lett. B 473, 241 (2000); D. Ida, J. High Energy Phys. 09 (2000) 014; P. Kraus, J. High Energy Phys. 12 (1999) 011.

[6] A. Hebecker and J. March-Russell, Nucl. Phys. B608, 375 (2001); D. Langlois, L. Sorbo, and M. RodriguezMartinez, Phys. Rev. Lett. 89, 171301 (2002); D. Langlois and L. Sorbo, Phys. Rev. D 68, 084006 (2003); E. Leeper, R. Maartens, and C.F. Sopuerta, Classical 
Quantum Gravity 21, 1125 (2004); K. Ichiki, M. Yahiro, T. Kajino, M. Orito, and G. J. Mathews, Phys. Rev. D 66, 043521 (2002).

[7] J. Garriga and M. Sasaki, Phys. Rev. D 62, 043523 (2000).

[8] S. W. Hawking, T. Hertog, and H. S. Reall, Phys. Rev. D 62, 043501 (2000).

[9] S. Kobayashi, K. Koyama, and J. Soda, Phys. Lett. B 501, 157 (2001); Y. Himemoto and M. Sasaki, Phys. Rev. D 63, 044015 (2001); Y. Himemoto, T. Tanaka, and M. Sasaki, Phys. Rev. D 65, 104020 (2002); T. Tanaka and Y. Himemoto, Phys. Rev. D 67, 104007 (2003).

[10] K. Koyama and K. Takahashi, Phys. Rev. D 67, 103503 (2003); K. Koyama and K. Takahashi, Phys. Rev. D 68, 103512 (2003); T. Kobayashi and T. Tanaka, Phys. Rev. D 69, 064037 (2004).

[11] R. Maartens, D. Wands, B. A. Bassett, and I. Heard, Phys. Rev. D 62, 041301 (2000); S. Mukohyama, Phys. Rev. D 62, 084015 (2000); H. Kodama, A. Ishibashi, and O. Seto, Phys. Rev. D 62, 064022 (2000); D. Langlois, Phys. Rev. D 62, 126012 (2000); D. Langlois, Phys. Rev. Lett. 86, 2212 (2001); H. A. Bridgman, K. A. Malik, and D. Wands, Phys. Rev. D 63, 084012 (2001); D. Langlois, R. Maartens, M. Sasaki, and D. Wands, Phys. Rev. D 63, 084009 (2001); H. A. Bridgman, K. A. Malik, and D. Wands, Phys. Rev. D 65, 043502 (2002); K. Koyama and J. Soda, Phys. Rev. D 62, 123502 (2000); 65, 023514 (2002); B. Gumjudpai, R. Maartens, and C. Gordon, Classical Quantum Gravity 20, 3295 (2003).

[12] H. Kodama and M. Sasaki, Prog. Theor. Phys. Suppl. 78, 1 (1984).

[13] V.F. Mukhanov, H. A. Feldman, and R. H. Brandenberger, Phys. Rep. 215, 203 (1992).

[14] K. Koyama, Phys. Rev. D 66, 084003 (2002).

[15] K. Koyama, Phys. Rev. Lett. 91, 221301 (2003).

[16] C. S. Rhodes, C. van de Bruck, P. Brax, and A. C. Davis, Phys. Rev. D 68, 083511 (2003).
[17] K. Koyama, J. Cosmol. Astropart. Phys. 09 (2004) 010 .

[18] K. Koyama, D. Langlois, R. Maartens, and D. Wands, J. Cosmol. Astropart. Phys. 11 (2004) 002.

[19] H. Yoshiguchi and K. Koyama, Phys. Rev. D 71, 043519 (2005).

[20] D. Langlois, R. Maartens, and D. Wands, Phys. Lett. B 489, 259 (2000).

[21] D.S. Gorbunov, V.A. Rubakov, and S. M. Sibiryakov, J. High Energy Phys. 10 (2001) 015.

[22] T. Kobayashi, H. Kudoh, and T. Tanaka, Phys. Rev. D 68, 044025 (2003).

[23] T. Hiramatsu, K. Koyama, and A. Taruya, Phys. Lett. B 578, 269 (2004).

[24] T. Hiramatsu, K. Koyama, and A. Taruya, Phys. Lett. B 609, 133 (2005).

[25] K. Ichiki and K. Nakamura, Phys. Rev. D 70, 064017 (2004).

[26] K. Ichiki and K. Nakamura, astro-ph/0406606.

[27] R. Easther, D. Langlois, R. Maartens, and D. Wands, J. Cosmol. Astropart. Phys. 10 (2003) 014.

[28] R. A. Battye, C. Van de Bruck, and A. Mennim, Phys. Rev. D 69, 064040 (2004).

[29] R. A. Battye and A. Mennim, Phys. Rev. D 70, 124008 (2004).

[30] T. Tanaka, gr-qc/0402068.

[31] T. Kobayashi and T. Tanaka, J. Cosmol. Astropart. Phys. 10 (2004) 015

[32] J.C. Long, H. W. Chan, A. B. Churnside, E. A. Gulbis, M. C. M. Varney, and J. C. Price, hep-ph/0210004.

[33] A. R. Liddle and D. H. Lyth, Cosmological Inflation and Large-Scale Structure (Cambridge University Press, Cambridge, England, 2000).

[34] In the $k^{2} \rightarrow 0$ limit, $\phi=$ const is a growing solution of Eq. (46). 